\title{
Preface
}

\section{Meniscus Injuries in Athletes}

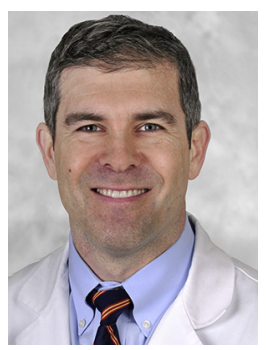

Brett D. Owens, MD

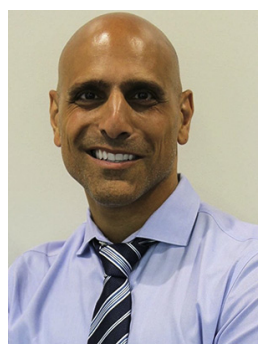

Ramin R. Tabaddor, MD

Editors

While so much of the sports medicine literature focuses on the anterior cruciate ligament (ACL), it is actually the meniscus that may be considered the critical player in the athletic knee. Much of the reasoning behind early ACL reconstruction is for preservation of the meniscus. Modern day cohorts of ACL reconstruction patients, such as the MOON cohort, continually show that it is the meniscal status that determines the athlete's overall outcome, and not all the reconstructive variables that receive so much of the surgeon's focus.

Once considered a vestigial organ, the meniscus plays a central role in knee mechanics, as both stabilizer, load distributor, and absorber. It is essential for the sports medicine clinician and knee surgeon to rule out the meniscus as a pain generator in the athlete's knee. Repair of meniscus tears remains central to the surgical treatment of athletic knee injuries. As new tear patterns become increasingly appreciated, advancing technical solutions augment the surgeon's armamentarium. Rehabilitation of these repairs has continued to advance as well, decreasing postoperative restrictions and improving active recovery. There has also been a heightened focus on return to play considerations, and this has continued to advance our field.

In this issue, we have brought together an international panel of experts on the meniscus as it relates specifically to the fields of epidemiology, cellular and tissue biology, surgical treatment, rehabilitation, and the care of athletes. We have attempted to comprehensively cover the topic of meniscus injury in athletes from epidemiology through return to play, with a large focus on surgical techniques. We added an article on basic science of meniscal repair due to its increasing relevance, given that the augmentation of repairs with biological adjuncts is becoming common. We hope you find this issue to be helpful in your care of the athletic knee, and we hope we 
xvi Preface

can stimulate further dialogue and inquiry on this essential topic, because we have learned, "As goes the meniscus, goes the knee...."

Brett D. Owens, MD Department of Orthopedics Brown University

Providence, RI 02912, USA

1 Kettle Point Avenue East Providence, RI 02914, USA

Ramin R. Tabaddor, MD Department of Orthopedics Brown University Providence, RI 02912, USA

1 Kettle Point Avenue East Providence, RI 02914, USA

E-mail addresses: owensbrett@gmail.com (B.D. Owens) rontabaddor@gmail.com (R.R. Tabaddor) 